\title{
A GEOMETRIC PROPERTY OF CONVEX SETS WITH APPLICATIONS TO MINIMAX TYPE INEQUALITIES AND FIXED POINT THEOREMS
}

\author{
MAU-HSIANG SHIH and KOK-KEONG TAN
}

(Received 14 April 1986; revised 31 August 1986)

Communicated by J. H. Rubinstein

\begin{abstract}
A geometric property of convex sets which is equivalent to a minimax inequality of the Ky Fan type is formulated. This property is used directly to prove minimax inequalities of the von Neumann type, minimax inequalities of the Fan-Kneser type, and fixed point theorems for inward and outward maps.

1980 Mathematics subject classification (Amer. Math. Soc.): primary 52 A 07, 49 A 40; secondary $47 \mathrm{H} 10$.

Keywords and phrases: minimax inequality, fixed point, inward and outward maps, partition of unity, convex set, topological vector space.
\end{abstract}

\section{Introduction}

Properties of convex sets in topological vector spaces related to fixed point and minimax theorems were given in Fan $[7,10-15]$. In 1972, Fan [13, Theorem 2] proved the following geometric theorem of convex sets which has numerous connections with other areas of mathematics and serves to unify many apparently diverse mathematical phenomena.

THEOREM 1 [KY FAN]. Let $X$ be a non-empty compact convex subset of a Hausdorff topological vector space and let $B \subset X \times X$. Assume

(a) For each fixed $x \in X$, the section $\{y \in X:(x, y) \in B\}$ is open in $X$.

This work was partially supported by NSERC of Canada under Grant A8096.

(C) 1988 Australian Mathematical Society 0263-6115/88 \$A2.00 + 0.00 
(b) For each fixed $y \in X$, the section $\{x \in X:(x, y) \in B\}$ is non-empty and convex.

Then there exists a point $x_{0} \in X$ such that $\left(x_{0}, x_{0}\right) \in B$.

In the present paper we shall extend Theorem 1 by relaxing the compactness and convexity conditions. Direct applications to minimax type inequalities and fixed point theorems are illustrated.

\section{A Geometric property of convex sets}

THEOREM 2. Let $X$ be a non-empty convex subset of a Hausdorff topological vector space and let $A, B \subset X \times X$. Assume

(a) For each fixed $x \in X$, the section $\{y \in X:(x, y) \in A\}$ is open in $X$.

(b) For each fixed $y \in X$, the section $\{x \in X:(x, y) \in B\}$ contains the convex hull of the section $\{x \in X:(x, y) \in A\}$.

(c) There exist a non-empty compact convex subset $X_{0}$ and a non-empty compact subset $K$ of $X$ such that

$\left(c_{1}\right)$ the section $\{x \in X:(x, y) \in A\} \neq \varnothing$ for all $y \in K$ and

(c) $X_{0} \cap\{x \in X:(x, y) \in A\} \neq \varnothing$ for all $y \in X \backslash K$.

Then there exists a point $x_{0} \in X$ such that $\left(x_{0}, x_{0}\right) \in B$.

ProOF. For each $x \in X$, let $A(x)=\{y \in X:(x, y) \in A\}$; then by (a), $A(x)$ is open in $X$ for each $x \in X$. By $\left(c_{1}\right), K \subset \bigcup_{x \in X} A(x)$. By compactness of $K$, there exists $\left\{x_{1}, x_{2}, \ldots, x_{n}\right\} \subset X$ such that

$$
K \subset \bigcup_{i=1}^{n} A\left(x_{i}\right) .
$$

Let $\Omega$ be the convex hull of $X_{0} \cup\left\{x_{1}, x_{2}, \ldots, x_{n}\right\}$ and define

$$
\begin{aligned}
& \tilde{A}=A \cap(\Omega \times \Omega), \\
& \tilde{B}=B \cap(\Omega \times \Omega) .
\end{aligned}
$$

Then $\Omega$ is a compact convex subset of $X$ and we have:

(i) For each fixed $x \in \Omega$, the section $\{y \in \Omega:(x, y) \in \tilde{A}\}$ is open in $\Omega$ by (a).

(ii) For each fixed $y \in \Omega$, the section $\{x \in \Omega:(x, y) \in \tilde{B}\}$ contains the convex hull of the section $\{x \in \Omega:(x, y) \in \tilde{A}\}$ by (b).

(iii) For each fixed $y \in \Omega$, the section $\{x \in \Omega:(x, y) \in \tilde{A}\} \neq \varnothing$ by $\left(c_{1}\right),\left(c_{2}\right)$ and $(*)$. 
Now, for each $x \in \Omega$, let $\tilde{A}(x)=\{y \in \Omega:(x, y) \in \tilde{A}\}$; then by (i) $\tilde{A}(x)$ is open in $\Omega$ for each $x \in \Omega$. By (iii), $\Omega=\bigcup_{x \in \Omega} \tilde{A}(x)$. By compactness of $\Omega$, there exists $\left\{y_{1}, y_{2}, \ldots, y_{m}\right\} \subset \Omega$ such that

$$
\Omega=\bigcup_{j=1}^{m} \tilde{A}\left(y_{j}\right) .
$$

Let $\left\{\alpha_{1}, \alpha_{2}, \ldots, \alpha_{m}\right\}$ be a partition of unity subordinate to the covering $\left\{\tilde{A}\left(y_{1}\right), \tilde{A}\left(y_{2}\right), \ldots, \tilde{A}\left(y_{m}\right)\right\}$. Thus, $\alpha_{1}, \alpha_{2}, \ldots, \alpha_{m}$ are continuous non-negative functions on $\Omega$ such that for each $j=1,2, \ldots, m, \operatorname{supp} \alpha_{j} \subset \tilde{A}\left(y_{j}\right)$ and

$$
\sum_{j=1}^{m} \alpha_{j}(y)=1 \quad \text { for all } y \in \Omega \text {. }
$$

Define $p: \Omega \rightarrow \Omega$ by setting

$$
p(y)=\sum_{j=1}^{m} \alpha_{j}(y) y_{j} .
$$

Then $p$ is a continuous map which maps the convex hull $\operatorname{conv}\left\{y_{1}, y_{2}, \ldots, y_{m}\right\}$ of $\left\{y_{1}, y_{2}, \ldots, y_{m}\right\}$ into itself. By Brouwer's fixed point theorem, there exists a point $x_{0} \in \operatorname{conv}\left\{y_{1}, y_{2}, \ldots, y_{m}\right\}$ such that $p\left(x_{0}\right)=x_{0}$. Note that for each $j=1,2, \ldots, m$, if $\alpha_{j}\left(x_{0}\right)>0$, then $x_{0} \in \tilde{A}\left(y_{j}\right)$ so that $\left(y_{j}, x_{0}\right) \in \tilde{A}$; it follows from (ii) that $\left(p\left(x_{0}\right), x_{0}\right) \in \tilde{B}$. This proves the theorem.

REMARKs. (1) When $A=B, X=X_{0}=K$, Theorem 2 reduces to Theorem 1. (2) When $A=B, X_{0}=K$, Theorem 2 still contains a theorem of Fan [14, Theorem 10]. (3) When $X=X_{0}=K$, Theorem 2 reduces to our earlier formulation [22, Theorem 3]. (4) Theorem 2 is equivalent to the following:

THEOREM 2'. Same hypotheses and conclusions as in Theorem 2 except that the condition (b) is replaced by $\left(\mathrm{b}_{1}\right) A \subset B$, and $\left(\mathrm{b}_{2}\right)$ for each fixed $y \in X$, the section $\{x \in X:(x, y) \in B\}$ is convex.

Theorem 2 has the following analytic form.

THEOREM 3. Let $X$ be a non-empty convex subset of a Hausdorff topological vector space and let $f$ and $g$ be two real-valued functions on $X \times X$. Assume

(a) $g(x, x) \leq 0$ for all $x \in X$.

(b) For each fixed $x \in X, f(x, y)$ is a lower semi-continuous function of $y$ on $X$.

(c) For each fixed $y \in X$, the set $\{x \in X: g(x, y)>0\}$ contains the convex hull of the set $\{x \in X: f(x, y)>0\}$. 
(d) There exists a non-empty compact convex subset $X_{0}$ of $X$ such that the set $\left\{y \in X: f(x, y) \leq 0\right.$ for all $\left.x \in X_{0}\right\}$ is compact.

Then there exists a point $\tilde{y} \in X$ such that $f(x, \tilde{y}) \leq 0$ for all $x \in X$.

Indication of a proof for the equivalence of Theorems 2 and 3 :

Theorem $2 \Rightarrow$ Theorem 3 . Let

$$
\begin{aligned}
& A=\{(x, y) \in X \times X: f(x, y)>0\} \\
& B=\{(x, y) \in X \times X: g(x, y)>0\} \\
& K=\left\{y \in X: f(x, y) \leq 0 \text { for all } x \in X_{0}\right\}
\end{aligned}
$$

and apply Theorem 2 .

Theorem $3 \Rightarrow$ Theorem 2. Let $f$ and $g$ be characteristic functions of $A$ and $B$, respectively, and apply Theorem 3 .

REMARKS. (1) When $f \equiv g, X=X_{0}=K$, Theorem 3 reduces to the wellknown Ky Fan minimax principle [13]. (2) When $f \equiv g$, Theorem 3 reduces to Fan's theorem [15, Theorem 6]. (3) When $f \equiv g$ and $X_{0}=K$, Theorem 3 reduces to Allen's theorem [1, Theorem 2]. (4) When $X_{0}=K$, Theorem 3 reduces to Tan's theorem [26, Theorem 1]. (5) When $X=X_{0}=K$, Theorem 3 reduces to Yen's theorem [27]. (6) Conditions (a) and (b) imply the set $\{y \in X: f(x, y) \leq 0$ for all $\left.x \in X_{0}\right\}$ is non-empty. The coercive condition (d) is a unification of the two coercive conditions given in Allen [1, Theorem 2, condition (d)] and in Brézis-Nirenberg-Stampacchia [3, Theorem 1, condition (5)].

The following example shows that Allen's theorem [1, Theorem 2] is properly contained in Fan's theorem [15, Theorem 6].

EXAMPLE. Let $0<p<1$,

$$
l_{p}=\left\{x=(x(n))_{n=1}^{\infty}: \sum_{n=1}^{\infty}|x(n)|^{p}<\infty\right\},
$$

$$
d_{p}(x, y)=\sum_{n=1}^{\infty}|x(n)-y(n)|^{p}, \quad \text { for all } x=(x(n))_{n=1}^{\infty}, y=(y(n))_{n=1}^{\infty} \in l_{p} .
$$

Then $\left(l_{p}, d_{p}\right)$ is a completely metrizable topological vector space which is not locally convex. Let $\left(x_{n}\right)_{n=0}^{\infty}$ be a sequence in $l_{p}$ defined by

$$
x_{0}=(0,0, \ldots), \quad x_{n}(k)= \begin{cases}0, & \text { if } k \neq n, \\ \frac{1}{n^{1-p}}, & \text { if } k=n\end{cases}
$$


Let $K=\left\{x_{n}: n=0,1,2, \ldots\right\}, X=\operatorname{conv}(K)$, the convex hull of $K$. Since $x_{n} \rightarrow 0$ as $n \rightarrow \infty, K$ is compact. Define $f: X \times X \rightarrow \mathbf{R}$ by

$$
\begin{aligned}
f(x, y) & =0 \quad \text { for each }(x, y) \in X \times X \text { with } x \neq 0, \\
f\left(0, \alpha x_{2 n}\right) & =0 \quad \text { for each } n=0,1,2, \ldots \text { and for each } \alpha \in[0,1], \\
f\left(0, \alpha x_{2 n+1}\right) & =\frac{1}{2 n+1} \quad \text { for each } n=0,1,2, \ldots \text { and for each } \alpha \in(0,1], \\
f\left(0, \sum_{i=1}^{N} \alpha_{i} x_{n_{i}}\right) & =N \quad \text { for each } N \geq 2, \text { for each } \alpha_{1}, \alpha_{2}, \ldots, \alpha_{N} \in(0,1] \\
& \text { with } 1 \leq n_{1}<n_{2}<\cdots<n_{N} \text { such that } \sum_{i=1}^{N} \alpha_{i} \leq 1 .
\end{aligned}
$$

I. For each fixed $x \in X, y \mapsto f(x, y)$ is lower semi-continuous.

Let $\lambda \in \mathbf{R}$ be given.

Case 1. Suppose $x \neq 0$. Then the set

$$
\{y \in X: f(x, y) \leq \lambda\}= \begin{cases}\varnothing & \text { if } \lambda<0 \\ X & \text { if } \lambda \geq 0\end{cases}
$$

is open in $X$.

Case 2. Suppose $x=0$. Then we see that

$$
\{y \in X: f(0, y) \leq \lambda\}=\left\{\begin{array}{l}
\varnothing, \quad \text { if } \lambda<0, \\
A_{0}=\left\{\alpha x_{2 n}: n=0,1,2, \ldots, \alpha \in[0,1]\right\}, \quad \text { if } \lambda=0, \\
A_{0} \cup\left\{\beta x_{2 n+1}: n \geq N+1, \beta \in[0,1]\right\}, \\
\quad \text { if } \frac{1}{2 N+3} \leq \lambda<\frac{1}{2 N+1}, N=0,1,2, \ldots, \quad \text { if } 1 \leq \lambda<2, \\
\left\{\alpha x_{n}: n=0,1,2, \ldots, \alpha \in[0,1]\right\}, \\
A_{N}, \quad \text { if } 2 \leq N \leq \lambda<N+1,
\end{array}\right.
$$

where

$A_{N}=\left\{\sum_{i=1}^{N} \alpha_{i} x_{n_{i}}: 0 \leq n_{1}<n_{2}<\cdots<n_{N}, \alpha_{1}, \alpha_{2}, \ldots, \alpha_{N} \in[0,1], \sum_{i=1}^{N} \alpha_{i} \leq 1\right\}$.

Now, $A_{0}$ is compact, being the continuous image of the compact set $\left\{x_{2 n}: n=\right.$ $0,1,2, \ldots\} \times[0,1]$. Similarly $\left\{\beta x_{2 n+1}: n \geq N+1, \beta \in[0,1]\right\}$ and $\left\{\alpha x_{n}: n=\right.$ $0,1,2, \ldots, \alpha \in[0,1]\}$ are compact. To show $\{y \in X: f(0, y) \leq \lambda\}$ is closed in $X$, it remains to show that $A_{N}$ is closed in $X$ for $N \geq 2$; in fact, each $A_{N}$ is compact, since it is the continuous image of the compact set $P_{N} \times\left(\prod_{i=1}^{N} K\right)$ where $P_{N}=$ $\left\{\left(\lambda_{1}, \lambda_{2}, \ldots, \lambda_{N}\right) \in \mathbf{R}^{N}: \lambda_{i} \geq 0\right.$ for all $i=1,2, \ldots, N$ and $\left.\sum_{i=1}^{N} \lambda_{i} \leq 1\right\}$. 
II. For each fixed $y \in X, x \mapsto f(x, y)$ is quasi-concave. Let $\lambda \in \mathbf{R}$ be given.

Case 1. If $\lambda<0$ or $y=\alpha x_{2 n}, n=0,1,2, \ldots, \alpha \in(0,1]$, the set $\{x \in$ $X: f(x, y)>\lambda\}=X$ is convex.

Case 2. If $\lambda \geq 0$ and $y=\alpha x_{2 n+1}$ for $n=0,1,2, \ldots, \alpha \in(0,1]$, the set

$$
\{x \in X: f(x, y)>\lambda\}= \begin{cases}\{0\}, & \text { if } 0 \leq \lambda<\frac{1}{2 n+1}, \\ \varnothing, & \text { if } \lambda \geq \frac{1}{2 n+1},\end{cases}
$$

is also convex.

Case 3. If $\lambda \geq 0$ and $y=\sum_{i=1}^{N} \alpha_{i} x_{n_{i}}$ for $1 \leq n_{1}<\cdots<n_{N}, \alpha_{1}, \ldots, \alpha_{N} \in$ $(0,1)$ with $\sum_{i=1}^{N} \alpha_{i} \leq 1$ where $N \geq 2$,

$$
\{x \in X: f(x, y)>\lambda\}= \begin{cases}\{0\}, & \text { if } 0 \leq \lambda<N \\ \varnothing, & \text { if } \lambda \geq N\end{cases}
$$

is also convex.

III. Allen's coercive condition is not satisfied, i.e., there does not exist a nonempty compact convex subset $M$ of $X$ such that for each $y \in X \backslash M$, there exists $x \in M$ with $f(x, y)>0$.

Suppose the contrary, that is suppose there exists a non-empty compact convex subset $M$ of $X$ such that for each $y \in X \backslash M$, there exists $x \in M$ with $f(x, y)>0$. Since $f(x, y)=0$ for each $x, y \in X$ with $x \neq 0$, we must have $0 \in M$ and for all $y \in X \backslash M, f(0, y)>0$. As $f\left(0, x_{2 n}\right)=0$ for $n=0,1,2, \ldots,\left\{x_{2 n}: n=\right.$ $0,1,2, \ldots\} \subset M$ and hence conv $\left\{x_{2 n}: n=0,1,2, \ldots\right\} \subset M$ since $M$ is convex. We shall show that $\operatorname{conv}\left\{x_{2 n}: n=0,1,2, \ldots\right\}$ is unbounded. Indeed

$$
\begin{aligned}
d_{p}\left(0,1 / N \sum_{n=1}^{N} x_{2 n}\right) & =\sum_{n=1}^{N} 1 / N^{p} \cdot 1 /(2 n)^{(1-p) p} \\
& \geq N \cdot 1 / N^{p} \cdot 1 /(2 N)^{(1-p) p} \\
& =1 / 2^{p(1-p} \cdot N^{(1-p)^{2}} \rightarrow \infty \quad \text { as } N \rightarrow \infty .
\end{aligned}
$$

Therefore $\operatorname{conv}\left\{x_{2 n}: n=0,1,2, \ldots\right\}$ is an unbounded subset of the compact convex set $M$, which is impossible.

IV. Fan's coercive condition is satisfied, that is there exists a non-empty compact convex subset $X_{0}$ of $X$ such that the set $\left\{y \in X: f(x, y) \leq 0\right.$ for all $\left.x \in X_{0}\right\}$ is compact. 
Indeed, take $X_{0}=\{0\} ;$ then

$\left\{y \in X: f(x, y) \leq 0\right.$ for all $\left.x \in X_{0}\right\}=\left\{\alpha x_{2 n}: n=0,1,2, \ldots, \alpha \in[0,1]\right\}=A_{0}$

is compact.

\section{Minimax inequalities of the von Neumann type}

We have shown that Theorem 2 is equivalent to a minimax inequality of the Ky Fan type. We shall now show that Theorem 2 also implies minimax inequalities of the von Neumann type directly.

THEOREM 4. Let $X$ and $Y$ be non-empty convex sets, each in a Hausdorff topological vector space, and let $f, u, v, g$ be four real-valued functions on $X \times Y$. Assume

(a) $u \leq v$ on $X \times Y$. $Y$.

(b) For each fixed $x \in X, f(x, y)$ is a lower semi-continuous function of $y$ on

(c) For each fixed $y \in Y, g(x, y)$ is an upper semi-continuous function of $x$ on $X$.

(d) For each fixed $y \in Y$ and for each $\lambda \in \mathbf{R}$, the sectin $\{x \in X: u(x, y)>\lambda\}$ contains the convex hull of the section $\{x \in X: f(x, y)>\lambda\}$.

(e) For each fixed $x \in X$ and for each $\lambda \in \mathbf{R}$, the section $\{y \in Y: v(x, y)<\lambda\}$ contains the convex hull of the section $\{y \in Y: g(x, y)<\lambda\}$.

(f) For a given fixed $\rho \in \mathbf{R}$, suppose there exist a non-empty compact convex subset $X_{0}$ of $X \times Y$ and a non-empty compact subset $K$ of $X \times Y$ such that

$$
X_{0} \cap[\{w \in X: f(w, y)>\rho\} \times\{z \in Y: g(x, z)<\rho\}] \neq \varnothing
$$

for each $(x, y) \in(X \times Y) \backslash K$.

Then there exists a point $\left(x_{0}, y_{0}\right) \in K$ such that either $f\left(x, y_{0}\right) \leq \rho$ for all $x \in X$ or $g\left(x_{0}, y\right) \geq \rho$ for all $y \in Y$.

Proof. For each $(x, y) \in X \times Y$, let

$$
\begin{aligned}
C(y)=\{x \in X: f(x, y)>\rho\}, & & D(y)=\{x \in X: u(x, y)>\rho\}, \\
E(x)=\{y \in Y: v(x, y)<\rho\}, & & F(x)=\{y \in Y: g(x, y)<\rho\} .
\end{aligned}
$$

Define

$$
\begin{aligned}
& A=\bigcup_{(x, y) \in X \times Y} C(y) \times F(x) \times\{(x, y)\}, \\
& B=\bigcup_{(x, y) \in X \times Y} D(y) \times E(x) \times\{(x, y)\} .
\end{aligned}
$$


Suppose that the assertion of the theorem were false. Then for each point $(\bar{x}, \bar{y}) \in K$, there exists $(x, y) \in X \times Y$ such that $F(x, \bar{y})>\rho$ and $g(\bar{x}, y)<\rho$ so that

$$
\{(x, y) \in X \times Y:((x, y),(\bar{x}, \bar{y})) \in A\} \neq \varnothing \quad \text { for each }(\bar{x}, \bar{y}) \in K .
$$

By (f),

$X_{0} \cap\{(x, y) \in X \times Y:((x, y),(\bar{x}, \bar{y})) \in A\} \neq \varnothing \quad$ for each $(\bar{x}, \bar{y}) \in(X \times Y) \backslash K$.

Other conditions of Theorem 2 are easily derived from the hypotheses of Theorem 4. Thus, according to Theorem 2, there exists a point $\left(x_{0}, y_{0}\right) \in X \times Y$ such that $\left(\left(x_{0}, y_{0}\right),\left(x_{0}, y_{0}\right)\right) \in B$; it follows that

$$
\rho<u\left(x_{0}, y_{0}\right) \leq v\left(x_{0}, y_{0}\right)<\rho,
$$

which is a contradiction. This proves the theorem.

When $X$ and $Y$ are compact, the condition (f) in Theorem 4 is satisfied by settng $X_{0}=K=X \times Y$. Thus Theorem 4 is a generalization of a minimax inequality in [2, Theorem 5.4$]$ by relaxing the compactness and convexity conditions.

Theorem 4 implies the following:

THEOREM 5. Let $X$ and $Y$ be non-empty convex sets, each in a Hausdorff topological vector space, and let $f, u, v, g$ be four real-valued functions on $X \times Y$. Assume the conditions (a), (b), (c), (d), (e) in Theorem 4 are satisfied.

(1) If there exists non-empty compact convex sets $M_{0} \subset X, N_{0} \subset Y$ and there exist non-empty compact sets $M \subset X, N \subset Y$ such that $\inf _{y \in Y} \sup _{x \in M_{0}} f(x, y)$ $=\inf _{y \in N} \sup _{x \in X} f(x, y)$, and $\sup _{x \in X} \inf _{y \in N_{0}} g(x, y)=\sup _{x \in M} \inf _{y \in Y} g(x, y)$, then the following minimax inequality holds:

Inequality I: $\inf _{y \in N} \sup _{x \in X} f(x, y) \leq \sup _{x \in M} \inf _{y \in Y} g(x, y)$.

(2) If there exist non-empty compact convex sets $M_{0} \subset X$ and $N_{0} \subset Y$ such that $\inf _{y \in Y} \sup _{x \in M_{0}} f(x, y)=\inf _{y \in Y} \sup _{x \in X} f(x, y)$ and $\sup _{x \in X} \inf _{y \in N_{0}} g(x, y)$ $=\sup _{x \in X} \inf _{y \in Y} g(x, y)$ then the following minimax inequality holds:

Inequality II. inf $y \in Y \sup _{x \in X} f(x, y) \leq \sup _{x \in X} \inf _{y \in Y} g(x, y)$.

When $f \equiv u \equiv v \equiv g$, it is readily seen that Inequality I in Theorem 5 implies the following minimax equalities, which generalize the minimax principle of the von Neumann type due to Sion [19]:

$$
\begin{aligned}
& \min _{y \in N} \sup _{x \in X} f(x, y)=\max _{x \in M} \inf _{y \in Y} f(x, y), \\
& \inf _{y \in Y} \sup _{x \in X} f(x, y)=\sup _{x \in X} \inf _{y \in Y} f(x, y) .
\end{aligned}
$$

When $f \equiv u, v \equiv g$, Theorem 5 also contains a minimax inequality of Liu [19]. 


\section{Systems of convex inequalities}

According to Pietsch [21, page 40], a collection $\mathcal{F}$ of real-valued functions $f$ defined on a set $X$ is called concave if, given any finite subset $\left\{f_{1}, f_{2}, \ldots, f_{n}\right\}$ of $\mathcal{F}$ and $\alpha_{1}, \alpha_{2}, \ldots, \alpha_{n} \geq 0$ with $\sum_{i=1}^{n} \alpha_{i}=1$, there exists $f \in \mathcal{F}$ such that $f(x) \geq \sum_{i=1}^{n} \alpha_{i} f_{i}(x)$ for all $x \in X$.

The following theorem given in Pietsch's book [21, page 40] concerning systems of convex inequalities is useful to study absolutely $r$-summing operators [21, page 232], $(p, q)$-dominated operators [21, page 236] and absolutely $\tau$-summing operators [21, page 324].

THEOREM 6. Let $X$ be a non-empty compact convex subset of a Hausdorff topological vector space, and let $\mathcal{F}$ be a concave collection of lower semicontinuous convex real-valued functions $f$ on $X$. Suppose that for every $f \in \mathcal{F}$ there exists an $x \in X$ with $f(x) \leq \rho$. Then there exists a point $x_{0} \in X$ such that $f\left(x_{0}\right) \leq \rho$ for all $f \in \mathcal{F}$ simultaneously.

Observe that Theorem 6 is equivalent to a theorem of Fan $[9$, Theorem 1] and Pietsch referred Theorem 6 as Fan's Lemma. The proof of Theorem 6 in Pietsch's book used the well-known separation theorem on convex sets. Granas-Liu [16] obtained a result which is a generalization of Theorem 6 to three collections of functions whose proof used a minimax inequality of the von Neumann type. We shall use Theorem 2 (or equivalently, Theorem 3, which is a Ky Fan type minimax inequality) to further extend Theorem 6 .

Given any two collections $\mathcal{I}$ and $\mathcal{G}$ of real-valued functions on a set $X$, we shall write $\mathcal{F} \leq \mathcal{G}$ if for any $f \in \mathcal{F}$ there exists $g \in \mathcal{G}$ such that $f(x) \leq g(x)$ for all $x \in X$.

THEOREM 7. Let $X$ be a non-empty normal closed convex set in a Hausdorff topological vector space. Let $\mathcal{F}, \mathcal{G}$, and $\mathcal{H}$ be three collections of real valued functions on $X$ such that

(a) $\mathcal{F} \leq \mathcal{G} \leq \mathcal{H}$;

(b) for each $f \in \mathcal{F}, f$ is lower semi-continuous on $X$;

(c) for each $g \in \mathcal{G}, g$ is convex on $X$;

(d) the collection $H$ is concave;

(e) $X$ has a non-empty compact convex subset $X_{0}$ and a non-empty compact subset $K$ such that for any two finite sets $\left\{f_{1}, f_{2}, \ldots, f_{n}\right\} \subset \mathcal{F},\left\{g_{1}, g_{2}, \ldots, g_{n}\right\} \subset$ $\mathcal{G}$, for any $\alpha_{1}, \alpha_{2}, \ldots, \alpha_{n} \geq 0$ with $\sum_{i=1}^{n} \alpha_{i}=1$, and for any $y \in X \backslash K$ there 
exists $x \in X_{0}$ such that

$$
\sum_{i=1}^{n} \alpha_{i} f_{i}(y)>\sum_{i=1}^{n} \alpha_{i} g_{i}(x)
$$

Then given any $\rho \in \mathbf{R}$ one of the following properties holds.

(i) There is an $h \in \mathcal{H}$ such that $\inf _{x \in X} h(x)>\rho$.

(ii) There exists a point $\hat{y} \in K$ such that $f(\hat{y}) \leq \rho$ for all $f \in \mathcal{F}$.

ProOF. Without loss of generality we may assume that $\rho=0$. For each $f \in \mathcal{F}$, let $Q(f)=\{x \in K: f(x) \leq 0\}$; then $Q(f)$ is closed in $K$ by lower semicontinuity of $f$. If the set $\{Q(f): f \in \mathcal{F}\}$ has the finite intersection property, then by compactness of $K$ we obtain the alternative (ii). Suppose $\{Q(f): \in \mathcal{F}\}$ does not have the finite intersection property, then there are $f_{1}, f_{2}, \ldots, f_{n} \in \mathcal{F}$ such that $\bigcap_{i=1}^{n} Q\left(f_{i}\right)=\varnothing$. For each $i=1,2, \ldots, n$, let $V_{i}=X \backslash Q\left(f_{j}\right)$; then each $V_{i}$ is open in $X$ and $\left\{V_{1}, V_{2}, \ldots, V_{n}\right\}$ is an open covering of the normal space $X$. Let $\left\{\beta_{1}, \beta_{2}, \ldots, \beta_{n}\right\}$ be a continuous partition of unity subordinate to this open covering. Thus, $\beta_{1}, \beta_{2}, \ldots, \beta_{n}$ are continuous non-negative functions on $X$ such that for each $i=1,2, \ldots, n, \operatorname{supp} \beta_{i} \subset V_{i}$ and $\sum_{i=1}^{n} \beta_{i}(x)=1$ for $x \in X$. Choose $g_{1}, g_{2}, \ldots, g_{n} \in \mathcal{G}$ and $h_{1}, h_{2}, \ldots, h_{n} \in \mathcal{H}$ so that $f_{i} \leq g_{i} \leq h_{i}$ on $X$ for each $i=1,2, \ldots, n$. Define

$$
\begin{aligned}
& A=\left\{(x, y) \in X \times X: \sum_{i=1}^{n} \beta_{i}(y) f_{i}(y)>\sum_{i=1}^{n} \beta_{i}(y) g_{i}(x)\right\} \\
& B=\left\{(x, y) \in X \times X: \sum_{i=1}^{n} \beta_{i}(y) g_{i}(y)>\sum_{i=1}^{n} \beta_{i}(y) g_{i}(x)\right\}
\end{aligned}
$$

Then the conditions (a), (b), ( $\left.c_{2}\right)$ of Theorem 2 are satisfied. Since for each $x \in X,(x, x) \notin B$, by Theorem 2 , there exists $\hat{y} \in K$ such that $\{x \in X:(x, \hat{y}) \in$ $A\}=\varnothing$. Therefore

$$
\sum_{i=1}^{n} \beta_{i}(\hat{y}) f_{i}(\hat{y}) \leq \sum_{i=1}^{n} \beta_{i}(\hat{y}) g_{i}(x) \text { for all } x \in X .
$$

By concavity of $\mathcal{H}$, there is an $h \in \mathcal{H}$ satisfying $h(x) \geq \sum_{i=1}^{n} \beta_{i}(\hat{y}) h_{i}(x)$ for all $x \in X$. Consequently,

$$
\begin{aligned}
0 & <\sum_{i=1}^{n} \beta_{i}(\hat{y}) f_{i}(\hat{y}) \leq \sum_{i=1}^{n} \beta_{i}(\hat{y}) g_{i}(x) \\
& \leq \sum_{i=1}^{n} \beta_{i}(\hat{y}) h_{i}(x) \leq h(x) \quad \text { for all } x \in X .
\end{aligned}
$$

This proves the alternative (i). This completes the proof. 
In the case when $X$ is compact convex, condition (e) in Theorem 7 is satisfied by setting $X_{0}=K=X$. Thus Theorem 7 generalizes Granas-Liu's result [16].

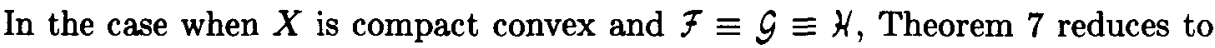
Theorem 6.

Let $h$ be a real-valued function defined on the product set $X \times Y$ of two arbitrary non-empty sets $X, Y$. According to Fan [8], $h$ is said to be concave on $X$, if for any two elements $x_{1}, x_{2} \in X$ and two numbers $\alpha_{1} \geq 0, \alpha_{2} \geq 0$ with $\alpha_{1}+\alpha_{2}=1$, there exists $x_{0} \in X$ such that

$$
h\left(x_{0}, y\right) \geq \alpha_{1} h\left(x_{1}, y\right)+\alpha_{2} h\left(x_{2}, y\right) \text { for all } y \in Y \text {. }
$$

THEOREM 8. Let $X$ be an arbitrary non-empty set and $Y$ a non-empty normal closed convex subset of a Hausdorff topological vector space. Let $f, g, h$ : $X \times Y \rightarrow \mathbf{R}$ be three functions such that

(a) $f \leq g \leq h$ on $X \times Y$;

(b) for each fixed $x \in X, f(x, y)$ is a lower semi-continuous function of $y$ on $Y$;

(c) for each fixed $x \in X, g(x, y)$ is a convex functions of $y$ on $Y$;

(d) $h$ is concave on $X$;

(e) $Y$ has a non-empty compact convex subset $X_{0}$ and a non-empty compact subset $K$ such that for each finite subset $\left\{x_{1}, x_{2}, \ldots, x_{n}\right\}$ of $X$, for any $\alpha_{1}, \alpha_{2}, \ldots, \alpha_{n} \geq 0$ with $\sum_{i=1}^{n} \alpha_{i}=1$, and for any $y \in Y \backslash K$ there exists $x \in X_{0}$ such that $\sum_{i=1}^{n} \alpha_{i} f\left(x_{i}, y\right)>\sum_{i=1}^{n} \alpha_{i} g\left(x_{i}, x\right)$.

Then

$$
\min _{y \in K} \sup _{x \in X} f(x, y) \leq \sup _{x \in X} \inf _{y \in Y} h(x, y)
$$

ProOF. Let $\rho=\sup _{x \in X} \inf _{y \in Y} h(x, y)$. Applying Theorem 7 with $X$ being the index set, there is a $\hat{y} \in K$ such that $f(x, \hat{y}) \leq \rho$ for all $x \in X$. The conclusion follows.

In Theorem $8, X$ is not required to possess any topological or linear structure. When $X$ is convex and $Y$ is compact convex, Theorem 8 is due to Granas-Liu [16]. The connection of Fan's convex inequalities with minimax theorems was pointed out by Takahashi [25].

When $f \equiv g \equiv h$, we obtain the following new minimax theorem.

THEOREM 9. Let $X$ be an arbitrary non-empty set and $Y$ a non-empty normal closed convex subset of a Hausdorff topological vector space. Let $f$ be $a$ real-valued function defined on $X \times Y$ such that

(a) For each fixed $x \in X, f(x, y)$ is a lower semi-continuous convex function of $y$ on $Y$;

(b) $f$ is concave on $X$; 
(c) $Y$ has a non-empty compact convex subset $X_{0}$ and a non-empty compact subset $K$ such that for each finite subset $\left\{x_{1}, x_{2}, \ldots x_{n}\right\}$ of $X$, for any $\alpha_{1}, \alpha_{2}, \ldots, \alpha_{n} \geq 0$ with $\sum_{i=1}^{n} \alpha_{i}=1$, and for any $y \in Y \backslash K$ there exists $x \in X_{0}$ such that

$$
\sum_{i=1}^{n} \alpha_{i} f\left(x_{i}, y\right)>\sum_{i=1}^{n} \alpha_{i} g\left(x_{i}, x\right)
$$

Then

$$
\min _{y \in K} \sup _{x \in X} f(x, y)=\sup _{x \in X} \inf _{y \in Y} f(x, y)
$$

When $X$ is convex and $Y$ is compact convex, Theorem 9 is a well-known minimax theorem of Kneser [18]. Another generalization of Kneser's minimax theorem was obtained by Fan [8] where both the linear structures of $X$ and $Y$ are eliminated.

\section{Fixed point theorems}

Fixed point theorems for inward or outward maps, and for single-valued or setvalued maps were studied by Browder $[4,5,6]$, Fan $[12,13,15]$ and Halpern and Bergman [17]. In this section, we shall apply Theorem 2 to give a generalization of Browder's recent fixed point theorem [6] to non-compact convex sets.

THEOREM 10. Let $X$ be a non-empty convex subset of a Hausdorff topological vector space $E$ and let $f: X \rightarrow E$ be a continuous map. Suppose that $p$ is a continuous real-valued function on $X \times E$ such that for all $x \in X, p(x, \cdot)$ is a convex function on $E$. Assume that there exist a non-empty compact convex subset $X_{0}$ of $X$ and a non-empty compact subset $K$ of $X$ such that

(a) For each $y \in K$ with $y \neq f(y)$, there exists $x \in y+\bigcup_{\lambda>0} \lambda(X-y)$ such that $p(y, x-f(y))<p(y, y-f(y))$.

(b) For each $y \in X \backslash K$ with $y \neq f(y)$, there exists $x \in y+\bigcup_{\lambda \geq 1} \lambda\left(X_{0}-y\right)$ such that $p(y, x-f(y))<p(y, y-f(y))$.

Then $f$ has a fixed point in $X$.

Proof. Suppose that $f$ has no fixed point in $X$. Define $A=\{(x, y) \in$ $X \times X: p(y, x-f(y))<p(y, y-f(y))\}$. Then (i) For each fixed $x \in X$, the section $\{y \in X:(x, y) \in A\}$ is open in $X$ by continuities of $f$ and $p$. (ii) For each fixed $y \in X$, the section $\{x \in X:(x, y) \in A\}$ is convex since $p(y, \cdot)$ is a convex function. (iii) By (a), for each $y \in K$ there exists $x_{0} \in$ $y+\bigcup_{\lambda>0} \lambda(X-y)$ such that $p\left(y, x_{0}-f(y)\right)<p(y, y-f(y))$. If $x_{0} \in X$, then the 
section $\{x \in X:(x, y) \in A\} \neq \varnothing$. If $x_{0} \notin X$, by convexity of $X$, there exist $\bar{x} \in X$ and $\lambda>1$ such that $x_{0}=y+\lambda(\bar{x}-y)$, so that $\bar{x}=((\lambda-1) / \lambda) y+(1 / \lambda) x_{0}$. As $p(y, \cdot)$ is convex, we have

$$
\begin{aligned}
p(y, \bar{x}-f(y)) & \leq \frac{\lambda-1}{\lambda} p(y, y-f(y))+\frac{1}{\lambda} p\left(y, x_{0}-f(y)\right) \\
& <p(y, y-f(y))
\end{aligned}
$$

thus $(\bar{x}, y) \in A$ and hence the section $\{x \in X:(x, y) \in A\} \neq \varnothing$. (iv) By (b), for each $y \in X \backslash K$, there exists $\bar{x} \in X_{0}$ and $\lambda \geq 1$ such that $x=y+\lambda(\bar{x}-y)$ and $p(y, x-f(y))<p(y, y-f(y))$. If $\lambda=1$, then $x=\bar{x}$, so that $(\bar{x}, y) \in A$. If $\lambda>1$ by the same argument as in (iii), we also have $(\bar{x}, y) \in A$. In both cases, we conclude that

$$
X_{0} \cap\{x \in X:(x, y) \in A\} \neq \varnothing .
$$

Applying Theorem 2 with $A \equiv B$, there exists $\hat{x} \in X$ such that $(\hat{x}, \hat{x}) \in A$, which is impossible. Thus $f$ has a fixed point $X$, completing the proof.

Theorem 10 generalizes Browder's fixed point theorem [6, Theorem 1] to noncompact convex sets. By setting $p(x, y)=\|y\|$ in Theorem 5 if the underlying space is a normed linear space, we have the following generalization of the Browder fixed point theorem [6, Corollary 1] and therefore a new generalization of the classical Schauder fixed point theorem.

COROLLARY 1. Let $X$ be a non-empty convex subset of a normed linear space $E$ and let $f: X \rightarrow E$ be a continuous map. Suppose that there exist a non-empty compact convex subset $X_{0}$ of $X$ and a non-empty compact subset $K$ of $X$ such that

(a) For each $y \in K, f(y)$ lies in the closure of $y+\bigcup_{\lambda>0} \lambda(X-y)$.

(b) For each $y \in X \backslash K, f(y)$ lies in the closure of $y+\bigcup_{\lambda \geq 1} \lambda\left(X_{0}-y\right)$. Then $f$ has a fixed point.

REMARKS. (1) Theorem 10 and Corollary 1 remain valid if in the unions $\bigcup_{\lambda>0}$ and $\bigcup_{\lambda \geq 1}$ in conditions (a) and (b) are replaced by $\bigcup_{\lambda<0}$ and $\bigcup_{\lambda \leq-1}$, respectively. (2) A more general version of Corollary 1 has been obtained in our recent paper [23]. 


\section{References}

[1] G. Allen, 'Variational inequalities, complementarity problems, and duality theorems', J. Math. Anal. Appl. 58 (1977), 1-10.

[2] H. Ben-El-Mechaiekh, P. Deguire and A. Granas, 'Points fixes et coincidences pour les fonctions multivoques II (Applications de type $\phi$ et $\phi^{*}$ )', C.R. Acad. Sci. Paris Sér. I Math. 295 (1982), 381-384.

[3] H. Brézis, L. Nirenberg and G. Stampacchia, 'A remark on Ky Fan's minimax principle', Boll. Un. Mat. Ital. 6 (1972), 293-300.

[4] F. E. Browder, 'A new generalization of the Schauder fixed point theorem', Math Ann. 174 (1967), 285-290.

[5] F. E. Browder, 'The fixed point theory of multi-valued mappings in topological vector spaces', Math. Ann. 177 (1968), 283-301.

[6] F. E. Browder, 'On a sharpened form of the Schauder fixed-point theorem', Proc. Nat. Acad. Sci. U.S.A 74 (1977), 4749-4751.

[7] K. Fan, 'Fixed-point and minimax theorems in locally convex topological linear spaces', Proc. Nat. Acad. Sci. U.S.A. 38 (1952), 121-126.

[8] K. Fan, 'Minimax theorems', Proc. Nat. Acad. Sci. U.S.A. 39 (1953), $42-47$.

[9] K. Fan, 'Existence theorems and extreme solutions for inequalities concerning convex functions or linear transformations', Math. Z. 68 (1957), 205-216.

[10] K. Fan, 'A generalization of Tychonoff's fixed point theorem', Math. Ann. 142 (1961), 305-310.

[11] K. Fan, 'Sur un théorème minimax', C.R. Acad. Sci. Paris Sér I 259 (1964), 3925-3928.

[12] K. Fan, 'Extensions of two fixed point theorems of F. E. Browder', Math. Z. 112 (1969), 234-240.

[13] K. Fan, 'A minimax inequality and applications', Inequalities III, Ed. O. Shisha, pp. 103113 (Academic Press, 1972).

[14] K. Fan, 'Fixed-point and related theorems for non-compact convex sets', Game theory and related topics, Eds. O. Moeschlin and D. Pallaschke, pp. 151-156 (North-Holland, 1979).

[15] K. Fan, 'Some properties of convex sets related to fixed point theorems', Math. Ann. 266 (1984), 519-537.

[16] A. Granas and F.-C. Liu, 'Remark on a theorem of Ky Fan concerning systems of inequalities', Bull. Inst. Math. Acad. Sinica 11 (1983), 639-643.

[17] B. Halpern and G. Bergman, 'A fixed point theorem for inward and outward maps', Trans. Amer. Math. Soc. 130 (1968), 353-358.

[18] H. Kneser, 'Sur un théorèmé fondamental de la théorie des jeux', C.R. Acad. Sci. Paris 234 (1952), 2418-2420.

[19] F. C. Liu, 'A note on the von Neumann-Sion minimax principle', Bull. Inst. Math. Acad. Sinica 6 (1978), 517-524.

[20] J. von Neumann, 'Zur theorie der gesellschaftsspiele', Math. Ann. 100 (1928), 295-320.

[21] A. Pietsch, Operator ideals (North-Holland, Amsterdam, 1980).

[22] M.-H. Shih and K-K. Tan, 'The Ky Fan minimax principle, sets with convex sections, and variational inequalities', Differential geometry-calculus of variations and their applications Eds. M. Rassias and T. Rassias, pp. 471-481, a volume dedicated to the memory of L. Euler on the occasion of the 200th anniversary since his death, (Dekker, New York, 1985).

[23] M.-H. Shih and K.-K. Tan, 'Covering theorems of convex sets related to fixed-point theorems', Nonlinear and convex analysis: Proceedings in honor of Ky Fan, Eds. B.-L. Lin and S. Simons, pp. 235-244, (Dekker, 1987).

[24] M. Sion, 'On general minimax theorems', Pacific J. Math. 8 (1958), 171-176.

[25] W. Takahashi, Nonlinear variational inequalities and fixed point theorems, J. Math. Soc. Japan 28 (1976), 168-181. 
[26] K.-K. Tan, 'Comparison theorems on minimax inequalities, variational inequalities, and fixed point theorems', J. London Math. Soc. 23 (1983), 555-562.

[27] C. L. Yen, 'A minimax inequality and its applications to variational inequalities', Pacific J. Math. 97 (1981), 477-481.

Department of Mathematics

Chung Yuan University

Chung-Li, Taiwan

Republic of China
Department of Mathematics, Statistics and Computing Science

Dalhousie University

Halifax, Nova Scotia B3H 3J5

Canada 